\title{
Article 8
}

\author{
Natural Images Contour Segmentation \\ Khairul Adilah Ahmad, Sharifah Lailee Syed Abdullah \\ Faculty of Computer and Mathematical Sciences \\ Universiti Teknologi MARA \\ Mahmod Othman \\ Department of Fundamental and Applied Sciences \\ Universiti Teknologi PETRONAS
}

\begin{abstract}
This paper, a combination of edge detection and contour based segmentation approach for object contour delineation is proposed. The proposed approach employs a new methodology for segmenting the fruit contour from the indoor and outdoor natural images more effectively. The overall process is carried out in five steps. The first step is to pre-process the image in order to convert the colour image to grayscale image. Second step is the adoption of Laplacian of Gaussian edge detection and a new corner template detection algorithm for adjustment of the pixels along the edge map in the interpolation process. Third step is the reconstruction process by implementing two morphology operators with embedded of inversion condition and dynamic threshold to preserve and reconstruct object contour. Fifth step is ground mask process in which the outputs of the inference obtained for each pixel is combined to a final segmented output, which provides a segmented foreground against the black background. This proposed algorithm is tested over 150 indoor and 40 outdoor fruit images in order to analyse its efficiency. From the experimental results, it has been observed that the proposed segmentation approach provides better segmentation accuracy of $100 \%$ in segmenting indoor and outdoor natural images. This algorithm also present a fully automatic model based system for segmenting fruit images of the natural environment.
\end{abstract}

Keywords: Edge detection, Contour Segmentation, Dynamic Threshold, Fruit, Natural Images,

\section{Introduction}

Segmentation is an extremely important phase in many applications of computer vision, which is characteristically related with pattern recognition problems. The essential goal of segmentation is to identify the object of interest in an image or to delineate the object, which are meaningful for a given application. False segmentation will cause degradation of the subsequent image analysis steps such as feature extraction and classification. Generally, segmentation is one the most difficult tasks in image processing where the goal is to detect the presence of object, to separate object from the scene background and to localise the object is space. It can be difficult for many reasons containing ambiguous illumination, ambivalent background and assorted appearance.

The purpose of this paper is to investigate and analyse an efficient method that can be employed to separate the fruit image into two different areas namely, the foreground area and the background area. The foreground area is the part of the image that contains the fruit and hence, provides the information about the fruit. The background area meanwhile contains the noises. The process of separating the image's foreground from the background areas is known as image segmentation. 


\section{Related Works}

The initial goal in almost all image processing fruit detection and segmentation approaches is to segment the different pixels which appear in image into two classes: fruits and background (soil leaves, stem and grass). Background removal is an essential stage, and it has to be done in an appropriate way to avoid any misclassification. Several methods have been developed for segmenting fruit images. The common segmentation technologies used for this purpose are: threshold-based segmentation, region-based and edge-based segmentation.

\section{i. $\quad$ Threshold}

The thresholding approach is a simple and easiest segmentation technique because it classifies pixels into two categories. Basically, there are three types of thresholding: Global thresholding, local thresholding and dynamic thresholding. Global threshold methods such as the one proposed by Otsu (Otsu, 1979) produced an optimal threshold value in order to split the image into two different areas. The critical problem of a single global thresholding is the choice of the threshold value to obtain robust segmentation result (Mahdi \& Alzubaidi, 2013). To overcome these drawbacks, local thresholding techniques have been proposed for segmentation which used different thresholds in input image (Huang, Gao, \& Cai, 2005). These techniques estimate or compute a different threshold for each pixel according to the greyscale information from the local neighbourhood of the pixel. The ability of the local thresholding therefore has the potential to resolve the problem due to ambiguous illumination conditions and ambivalent background. Then, in dynamic thresholding, each pixel was compared with each dynamic threshold computed from sliding a kernel over the input image. Global thresholding in general has the lowest adaptability to segment objects in natural environment followed by local thresholding and dynamic thresholding.

\section{ii. Region-based}

The region-based approach is a commonly used method for the region of interest extraction. Region-based technique is divided into two categories; region growing and merging and region splitting and merging (Du \& Sun, 2004). The main idea of region growing-and-merging was to start from a seed point and expand according to the growth rule around a pre-defined seed until it met the growth conditions. The key problems and difficulties of this method was in selecting the seed points. This technique that attempted to model regions using global information was usually not ideal for segmenting intensity inhomogeneity. The region-based technique was unpopular due to numerous limitations (Lin, Wang, Kang, \& Wang, 2012). The main challenge in using region-based for fruit contour segmentation was local minima due to the shading effects and presence of strong edges due to the ambivalent background. The algorithm was also computationally more complex thus increasing the computational time.

\section{iii. Edge-based}

The edge-based approach is the process to mark the points in an image at which the brightness intensity or texture changes sharply. The edges provided important visual information since they corresponded to major physical, photometrical or geometrical variations in images (Ferreira, Kiranyaz, \& Gabbouj, 2006; Sharma, Singh, \& Kaur, 2013) and was very efficient. Edges characterize object boundaries and were therefore useful for next process of objects in an image. It is a critically low-level operation of image processing because edges represent important information. Applying an edge detector to an image significantly reduces the amount of the data to be processed. The contour of the edges depended on many parameters in the image such as illumination condition (Setayesh, Zhang, \& Johnston, 2013), background characteristic (Gongal, Amatya, Karkee, Zhang, \& Lewis, 2015) and object properties or largescale shape phenotype (Karaletsos, Stegle, Dreyer, Winn, \& Borgwardt, 2012). . The edge 
map includes explicit information about the position, orientation and strength of each pixel of the image (Mahmod, Sharifah Lailee, Khairul Adilah, Mohd Nazari, \& Ab Razak, 2016).

\section{Material and method}

Computer vision process consists of image acquisition, image preprocessing, segmentation. However, the focus of this paper is on the segmentation technique which consists improved edge-based method and contour segmentation. Segmentation process is crucial because the quality of segmented image affects the results of feature extraction and classification task. In order to test the applicability of the proposed method, the Harumanis mango fruit images are chosen to further illustrate the contour segmentation. The Harumanis is chosen because its have similar or identical colour tones for young and ripe mangoes which is a challenge in terms of the similarity of the background to the object of interest (e.g. green fruit against green flora) and foreground complications (e.g. leaves and wrap paper that cross in front of the fruit, breaking its object contour).

\section{Image Acquisition}

For this study, 190 images of the Harumanis fruit were captured in natural environment including images taken from the grading house (indoor images) and images taken from the field (outdoor images) as reported in Table 1. A total 105 images were randomly selected from 150 indoor images which were used for the training data set. The other 45 indoor and 40 outdoor images were used as a testing data set.

Table 1: Acquired image

\begin{tabular}{lll}
\hline & Indoor image & Outdoor image \\
\hline Training & 105 & 0 \\
Testing & 45 & 40 \\
\hline Total & 150 & 40 \\
\hline
\end{tabular}

All the images were captured using a digital camera equipped on smart phones. The captured Harumanis images were saved in joint photographic expert group (JPEG) format with a resolution of 1624 X 2448 pixels and maintained in the native RGB (Red, Green and Blue) colour format. Then, image preprocessing involves conversion of RGB image into greyscale image. Greyscale images is a prerequisite because it makes further exploitation of the images more efficient and easy. This ensures less processing time and effectiveness of the algorithm to find edges.

\section{Image Segmentation}

The critical task of the computer vision system (CVS) is the recognition of the region of interest through segmentation process of Harumanis mango natural image. Image segmentation was conducted to remove the background area. This process was important to ensure that only the desired object was processed. The two major difficulties faced by the CVS were, non-uniform illumination condition and, the uncertain background which includes different background colors, leaves, stems and other fruits. The conducted segmentation process was intelligent enough to separate the region of interest from the natural images automatically. This was done by integrating improved edge-based algorithm with coner-template and new contour-based delineation (etCD). The diagram of the Edge-template and Contour Delineation (etCD) method for delineating object candidates is shown in Figure 1. 


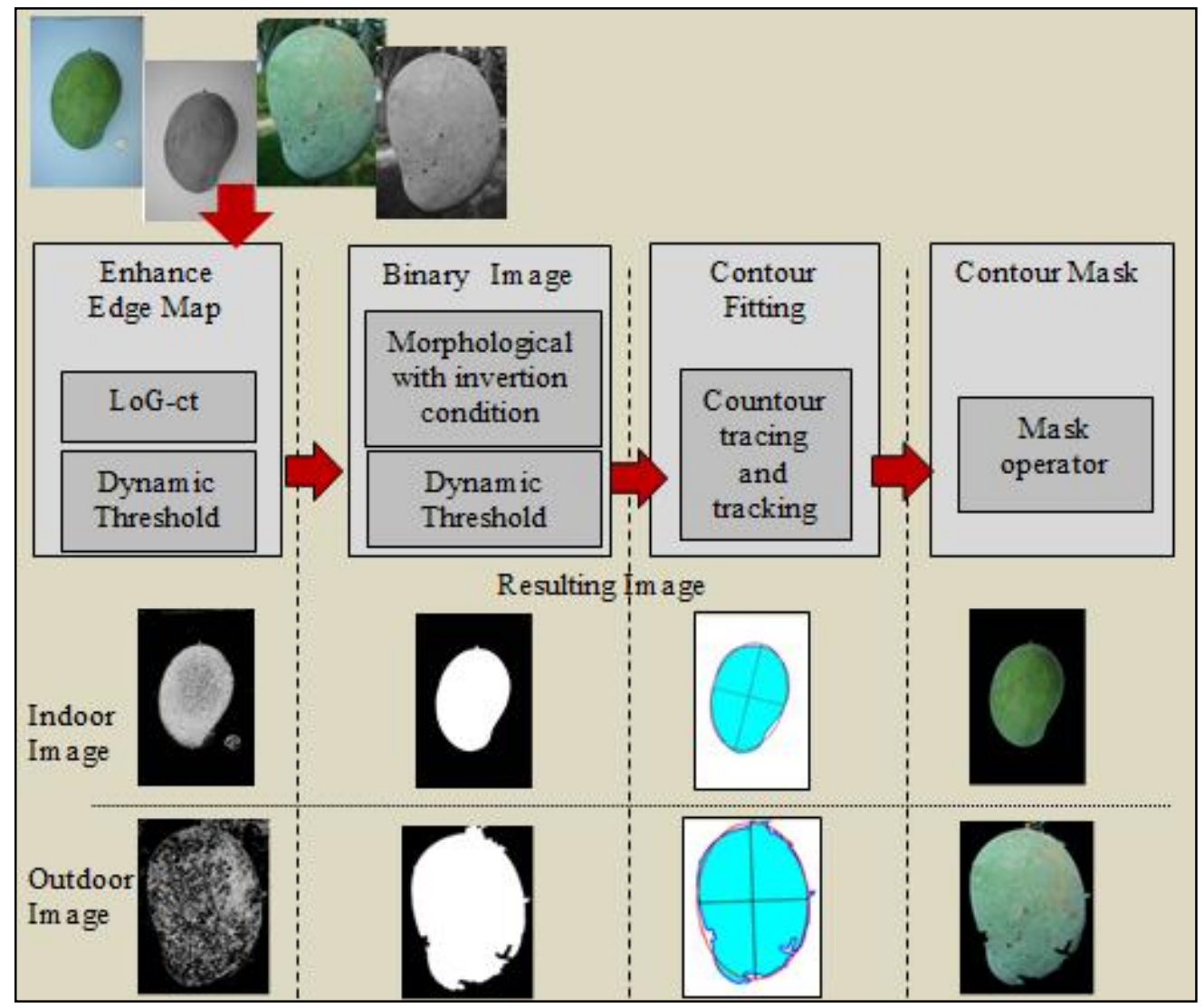

Figure 1: Diagram of the etCD Method

The etCD procedure consisted of four stages, enhances edge map, binary image, contour fitting and contour mask. The first part deals with improved edge detection by integrating Laplacian of Gaussian edge detector and new coner template to identify the fruit object candidate pixels. This improved the quality of edge map by enhancing the image and correcting the effect of non-uniform illumination. A dynamic blob area analysis thresholding algorithm is introduced to extract the mango images from an uncertain background. The algorithm eliminated the unwanted structure, hence preserving edge in uncontrolled acquisition conditions. In the degree of uncertainty in data points is high, then the amount of ambivalent in gradient image data will also be high, which will make the threshold identification is difficult. To solve the problem of ambivalent image this research proposes an algorithm using dynamic blob area analysis threshold to handle uncertainties that automatically selects the threshold value. A thresholding value based on the statistical principles was used to estimate the eligibility of edges to be an object in the image. The flowchart for dynamic blob area analysis threshold is shown in Figure 2. Finding the blobs are usually referred to as connected component analysis or connected component labelling (Grana, Borghesani, \& Cucchiara, 2009).

In the second stage, two morphology operators with embedded inversion condition and dynamic thresholds were implemented to preserve and reconstruct the robust fruit contour from various physical appearances. In this operation, a closing operator was used for fruit boundary smoothing and cleaning process by closing the holes in the contours of an object. Inversion condition was applied to identify the centre of the object candidate pixel. Inversion refers to the reverse process on each pixel of the binary image. If the pixel in the centre of the image pixel was black (' 0 '), an inverse process was executed to change the pixel value from ' 0 ' to ' 1 ', and vice versa. Next, a region filling operator was employed to fill in the holes within the fruit region. Finally, dynamic threshold was used to select the single object in the binary image. 
In the third stage, the single binary image was used to fit the appropriate ellipse into the labeled region. Since the shape of mangoes was ovoid, a direct least-squares ellipse fitting algorithm was used (Fitzgibbon, Pilu, \& Fisher, 1999) for the shape estimation. In the last stage, the grid of pixels was superimposed together with the initial segmentation map over the processed binary image. This image was processed using the contour-ground mask algorithm adapted from Sharifah Lailee, Hamirul'Aini, Khudzir, \& Nursuriati (2010).

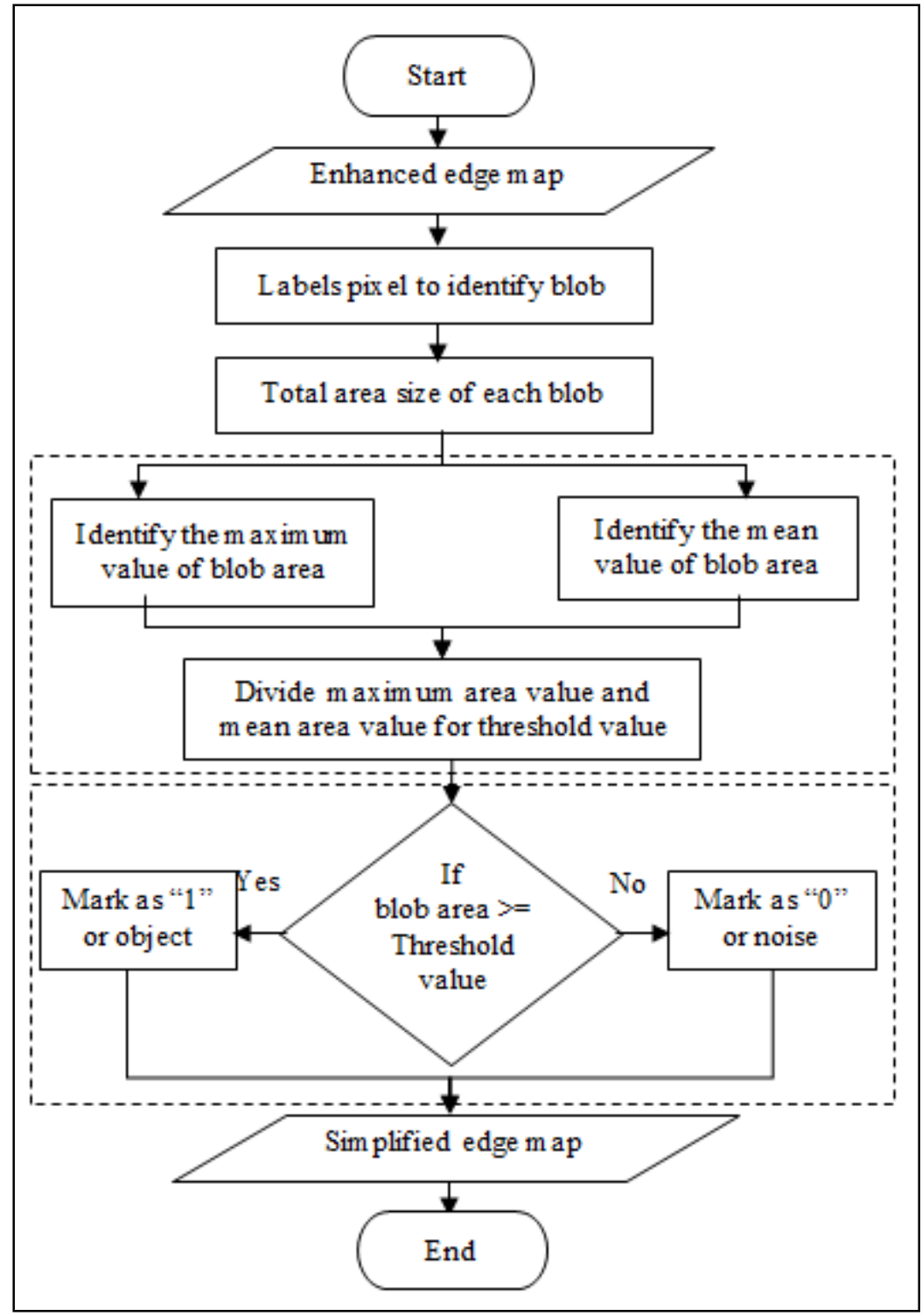

Figure 2: Flowchart of Dynamic Blob Area Analysis Threshold

\section{Results and Discussion}

In this research, a visual evaluation and quantitative evaluation method was used to show the performance of etCD algorithm. Visual evaluation was made based on human perception on the shape of the segmented image. For the quantitative evaluation method, a set of reference images called ground truth was created. The performance of etCD segmentations technique was evaluated by measuring the similarity and dissimilarity of the segmented images against the ground truth. The ground truth binary image dataset is a set of segmented images manually sketched by human and a quantitative performance measure was defined. In this research, a cropping technique was used to produce the ground truth dataset. Samples of the ground truth images are shown in Figure 3. 


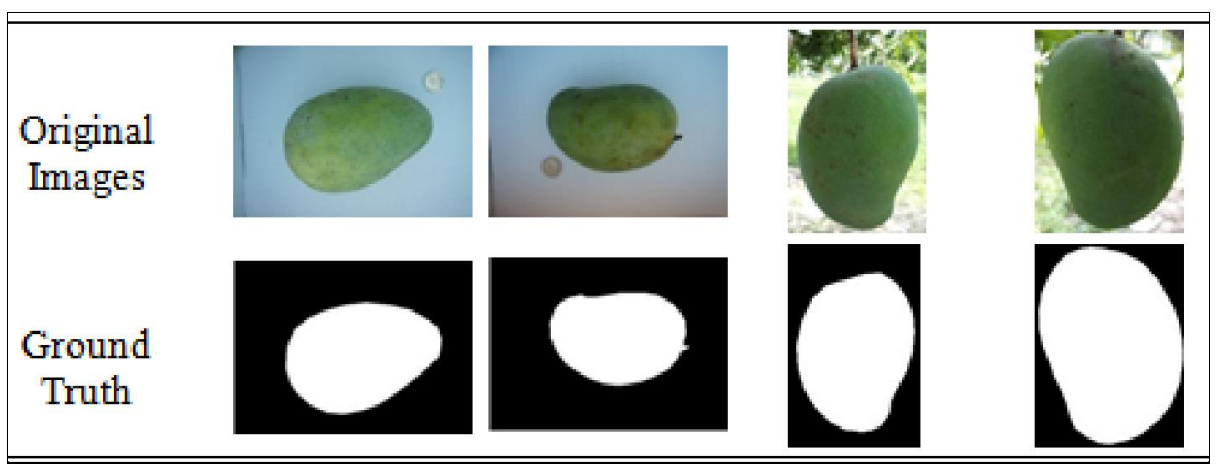

Figure 3: Original Images with Corresponding Ground Truth

For quantitative evaluation, two measurements were used in this research. The measurements were similarity index, Tanimoto Coefficient (TC) (Alaniz et al., 2006; Zijdenbos et al., 1994) and dissimilarity index, false positive rate (FPR) (Fawcett, 2006). The values of TC are between 0 and 1 in which 1 reflects a perfect similarity which indicates high quality of the segmented images.

The TC values measure the number of common pixels in the foreground and background areas shared by both segmented and ground truth images. Otherwise, the FPR known as oversegmentation was calculated when the pixels of the background were misclassified as foreground. The value of FPR also varies between 0 and 1 where the quality of segmented images is better when FPR values are lower.

The segmented results along with indoor ant outdoor images, Enhanced edge map, binary image, contour fitting, contour mask and its accuracy is given in Figure 4 and Figure 5 respectively.

\begin{tabular}{|c|c|c|c|c|}
\hline & $\mathrm{HI}$ & $\mathrm{H}_{2}$ & $\mathrm{H} 3$ & $\mathrm{H} 4$ \\
\hline \multicolumn{5}{|c|}{$\begin{array}{l}\text { Original } \\
\text { Image }\end{array}$} \\
\hline \multicolumn{5}{|c|}{$\begin{array}{l}\text { Enhanced } \\
\text { Edge map }\end{array}$} \\
\hline \multicolumn{5}{|c|}{$\begin{array}{l}\text { Binary } \\
\text { Image }\end{array}$} \\
\hline \multicolumn{5}{|c|}{$\begin{array}{l}\text { Contour } \\
\text { Fitting }\end{array}$} \\
\hline \multicolumn{5}{|c|}{$\begin{array}{l}\text { Contour } \\
\text { Mask }\end{array}$} \\
\hline $\mathrm{TC}$ & 0. & 0 & 0.99 & 0.994 \\
\hline FPR & 0.003 & 0.00 & 0.00 & 0.000 \\
\hline
\end{tabular}

Figure 4: Segmentation of Indoor Images using etCD 
For indoor images visual observation, the etCD algorithm has the ability to correctly segment the object contour with either strong or weak illumination exposure also fruit which contained foreground structure noise. In addition, the background areas of the segmented images were perfectly removed. Obviously, the segmentation visual results from the etCD algorithm were very accurate in segmenting fruit contour from indoor images. The TC values for all indoor images were high and almost near to the value of 1 , thus indicating that the etCD algorithm is able to produce perfect segmented for indoor images. Then, the evaluation of the algorithm result performed using FPR values were very small and almost near to the value of 1 . The smaller FPR values indicated etCD algorithm is able to remove the false positive of background pixels absolutely.

For outdoor images, in binary image, some small parts of the background areas in images F1 and F3 were wrongly classified because the background areas were filled with white pixels. The main factor for this misclassification of the background area was because the background object had similar intensity with the object area. Therefore, contour fitting was used to solve this problem. Contour fitting models was used to trace contour boundaries and to track the object contour using ellipse. Contour fitting has the advantage of naturally handle contour changes such as object over segmentation and therefore able to segment the object properly. Successful contour tracing and ellipse tracking has ignored the noise through circumnavigating the contour. he TC values for all the outdoor images produced by etCD algorithm were high and almost near the value of 1 . Moreover, the average value of 40 outdoor images TC is 0.937 , thus indicating that the algorithm is able to produce almost perfect segmented for outdoor images. The FPR values were very small which nearer to 0 . The lower FPR values also showed that there was less over segmentation of etCD algorithm.

\begin{tabular}{|l|llllllll}
\hline Original \\
Image
\end{tabular}

Figure 5: Segmentation of Outdoor Images using etCD 


\section{Conclusion}

In this paper a new method for segmenting fruit contour using the integration improved edgebased algorithm with coner-template and new contour-based delineation (etCD) algorithm had been proposed. The main purpose of this approach is to improve the accuracy of segmentation by reducing the segmentation error. The segmentation through edge detection and area analysis dynamic threshold found to reduce complex calculation which reduces the computation, which is very significant in real time processes. This method has been applied to 45 Harumanis indoor images and 40 Harumanis outdoor images in order to validate the efficiency of the etCD algorithm. In conclusion, the etCD algorithm has the ability to properly segment the contour of the object. Overall, all the segmented images produced by etCD have accurate object contour where all the pixels in the object were correctly filled with white pixels. In addition, the background area of the segmented images was mostly removed. Moreover, it proved to be robust in extracting object contour in the image in natural environment.

\section{References}

Ferreira, M., Kiranyaz, S., \& Gabbouj, M. (2006). A comparative analysis of feature extraction methods for fruit grading classifications. In IEEE International Conference on Acoustics Speed and Signal Processing Proceedings (Vol. 2, pp. 381-384).

Fitzgibbon, A., Pilu, M., \& Fisher, R. B. (1999). Direct least square fitting of ellipses. IEEE Transactions on Pattern Analysis and Machine Intelligence, 21(5), pp. 476-480.

Gongal, A., Amatya, S., Karkee, M., Zhang, Q., \& Lewis, K. (2015). Sensors and systems for fruit detection and localization: A review. Computers and Electronics in Agriculture, 116, pp. 8-19. h

Grana, C., Borghesani, D., \& Cucchiara, R. (2009). Connected component labeling techniques on modern architectures. In In Image Analysis and Processing-ICIAP, pp. 816-824. Springer Berlin Heidelberg.

Huang, Q., Gao, W., \& Cai, W. (2005). Thresholding technique with adaptive window selection for uneven lighting image. Pattern Recognition Letters, 26(6), pp. 801-808.

Karaletsos, T., Stegle, O., Dreyer, C., Winn, J., \& Borgwardt, K. M. (2012). ShapePheno: Unsupervised extraction of shape phenotypes from biological image collections. Bioinformatics, 28(7), pp. 1001-1008.

Lin, G.-C., Wang, W.-J., Kang, C.-C., \& Wang, C.-M. (2012). Multispectral MR images segmentation based on fuzzy knowledge and modified seeded region growing. Magnetic Resonance Imaging, 30(2), pp. 230-46.

Mahdi, A., \& Alzubaidi, N. (2013). Hybrid image segmentation method based on global thresholding method and edge detection using canny operator. Journal of Kerbala University, 11(3), pp. 63-74.

Mahmod, O., Sharifah Lailee, S. A., Khairul Adilah, A., Mohd Nazari, A. B., \& Ab Razak, M. (2016). The fusion of edge detection and mathematical morphology algorithm for shape boundary recognition. Journal of Information and Communication Technology (JICT), 15(1), pp. 133-144. 
Otsu, N. (1979). A threshold selection method from gray-level histograms. IEEE Transactions on Systems, Man, and Cybernetics, 9(1), pp. 62-66.

Setayesh, M., Zhang, M., \& Johnston, M. (2013). A novel particle swarm optimisation approach to detecting continuous, thin and smooth edges in noisy images. Information Sciences, 246, pp. 28-51.

Sharifah Lailee, S. A., Hamirul'Aini, H., Khudzir, I., \& Nursuriati, J. (2010). Improved technique for segmenting images under natural environment. International Conference on Science and Social Research, pp. 401-405.

Sharma, P., Singh, G., \& Kaur, A. (2013). Different techniques of edge detection in digital image processing. International Journal of Engineering Research and Applications, 3(3), pp. 458-461. 